\title{
3.9 SEARCHES FOR OPTICAL PULSARS
}

\author{
R. V. WILLSTROP \\ The Observatories, Madingley Road, Cambridge, U.K.
}

\begin{abstract}
The limitations of searches for pulsed optical radiation from pulsars and other objects are considered for the two cases (a) when the period is known from radio observations, and (b) when the period is unknown. Results of searches by the author and by others are summarised.
\end{abstract}

So far, radio searches for pulsars have been more successful than optical searches, by a factor of about 50 if we judge success by the number of positive identifications. However, in addition to measurements of NP 0532 many optical searches have established upper limits to the pulsed light flux reaching us from a number of other pulsars.

Let us consider the limitations of optical searches. The positions of some pulsars are already known within a few seconds of arc (Reichley et al., 1970), and some searches have been made using photometer diaphragms as small as 10 arc sec in diameter. Photon counting equipment is normally used. Because the optical emission from NP 0532 is a broad band phenomenon, photomultipliers are usually used without any filter in searches for other optical pulsars, and the light of the night sky causes many more counts than the dark emission of the detector. It is therefore the statistical fluctuations in the night sky light transmitted by the diaphragm which limit the sensitivity of optical searches.

It is easy to show that, with a multichannel scaler giving time resolution equal to the undispersed radio pulse duration, and supposing that an excess of detected photons at one phase amounting to five standard deviations is certain to be recognised, the minimum detectable pulsed photon flux outside the Earth's atmosphere is

$$
\mathscr{F}_{\text {min }}=5\left(\frac{N S f}{A T \varepsilon}\right)^{1 / 2} \text { photons } \mathrm{cm}^{-2} \mathrm{sec}^{-1}
$$

where $N$ photons $\mathrm{cm}^{-2} \mathrm{sec}^{-1}$ reach the Earth's atmosphere from each square second of arc of sky;

$S$ square seconds of arc of the sky are searched;

$f$ is the fraction of the pulsar period occupied by the undispersed radio pulse (the duty cycle);

$A \mathrm{~cm}^{2}$ is the collecting area of the telescope;

$T$ sec is the observing time used;

and $\varepsilon$ is the overall quantum efficiency, including the transmission of the atmosphere and telescope.

Clearly, searches to very faint limits depend on the determination of accurate positions by radio methods. 
At a reasonably dark observing site the sky brightness is about $21^{\mathrm{m}} .5$ per square second of arc, equivalent to $N=6 \times 10^{-3}$ photons $\mathrm{cm}^{-2} \mathrm{sec}^{-1}$ (arc sec) ${ }^{-2}$ in the spectral range 3500 to $6000 \mathrm{~A}$. Cocke and Disney (1970) searched recently for optical pulsars in the directions of CP 0328 and CP 0950 and claimed limiting sensitivities (apparent magnitudes) of $25^{\mathrm{m}} .5$ and $24^{\mathrm{m}} .5$ (later improved to $25^{\mathrm{m}} \cdot 6$ ). Equation (1) gives limits of 25.5 and 25.2 . It is reassuring to find such agreement between theory and practice.

Kristian reported (this symposium, Paper 2.3, p. 87) his observations of 11 pulsars, reaching limits between $20^{\mathrm{m}}$ and $25^{\mathrm{m}}$, all with negative results. Lynds et al. (1968) very early set a limit of $26^{\mathrm{m}}$ on CP 1919. Chiu (private communication) has searched the area around PSR $0833-46$ to a limit of $24^{\mathrm{m}}$, using an image intensifier to provide spatial resolution as well as time resolution. I have yet another negative observation to report, of HP 1506. Mrs. Mitton, one of the students at the Cambridge Observatories, pointed out (private communication) that the radio position is $1.4 \mathrm{sec}$ following and 7 arc sec south of a star of about $16^{\mathrm{m}}$. This coincidence seemed good enough to investigate further. A fainter star, about $19^{\mathrm{m}}, 16$ arc sec Nf the first, was also observed. The shading in Figure 1 represents the excess or deficiency in successive time bins, and the inset in each case shows the predicted effect of a $22^{\mathrm{m}}$ star with all its light pulsed at the period of HP 1506. Evidently the light of these two objects is not modulated by more than about 1 per cent and 10 per cent respectively.

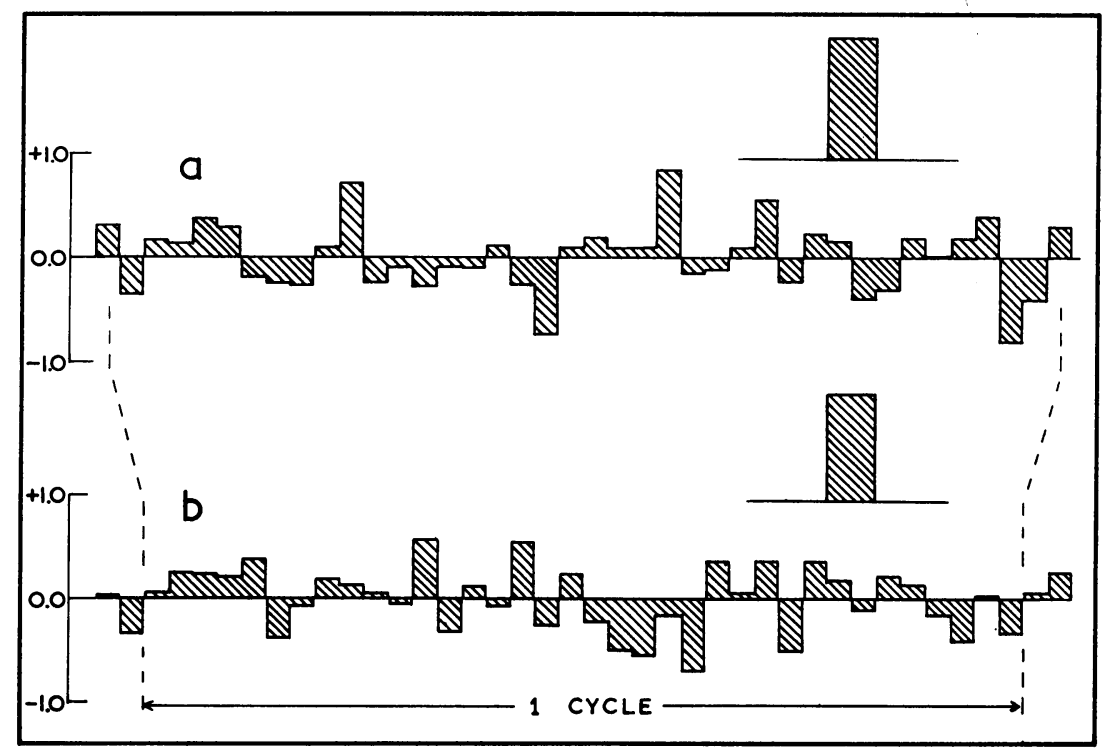

Fig. 1. Results of searches for optical variability in two stars near HP $1506\left(\alpha=15^{\mathrm{h}} 08^{\mathrm{m}} 03^{\mathrm{s}} .3\right.$ $\delta=+55^{\circ} 42^{\prime} 50^{\prime \prime}(1950)$ ). Ordinates: Variation in the rate of detection of photons from the star and sky, in per cent, as a function of phase. Abscissae: Phase, relative to an arbitrary origin. (a) 1970 June $322^{\mathrm{h}} 53^{\mathrm{m}}$ to $23^{\mathrm{h}} 30^{\mathrm{m}}: 52.072$ readings/sec. Summation over 3001 cycles. $16^{\mathrm{m}}$ star at $\alpha=15^{\mathrm{h}} 08^{\mathrm{m}} 01^{\mathrm{s}} .9$ $\delta=+55^{\circ} 42^{\prime} 57^{\prime \prime}$ (1950) (J. Mitton, private communication). (b) 1970 June $422^{\mathrm{h}} 57^{\mathrm{m}}$ to $23^{\mathrm{h}} 36^{\mathrm{m}}: 48.8176$ readings/sec. Summation over 3154 cycles. $19^{\mathrm{m}}$ star Nf the $16^{\mathrm{m}}$ star. In both observations the photometer diaphragm was 25 arc sec in diameter. 
I now wish to discuss the problem of detecting pulsed optical radiation in cases when no pulsed radio emission has been detected, and the period is therefore unknown. This problem arises because Cavaliere and Pacini (1970) have advocated a radio search for a pulsar associated with the supernova remnant Cas A, and Bahcall et al. (1970) have suggested optical and X-ray examination of newly reported supernovae nearer than $10 \mathrm{Mpc}$. The recent appearance of a supernova of $11^{\mathrm{m}}$ in $\mathrm{M} 101$ at $1.1 \mathrm{Mpc}$ adds to the interest of their suggestion. Signal averaging methods must be used, to overcome the statistical fluctuations in the night sky light, but the multichannel scaler is not a suitable recording device because it depends on the period being known. Instead, the numbers of photons detected in equal short intervals must be recorded on magnetic tape, paper tape or cards, and subsequently analysed, by computer, for all independent periods, for example by the Cooley-Tukey fast Fourier transform. Recording equipment of this type is available at the Hale Observatories, Harvard, Cambridge (U.K.), and some other observatories.

Figure 2 shows the plotter output from a recent analysis of my earliest data on the Crab Nebula, obtained on 24 November 1968. The plot is based on observations covering $2^{\mathrm{m}} 48^{\mathrm{s}}$ (16384 readings at 97.64 per sec) using a 36 -inch telescope with a diaphragm 50 arc sec in diameter, in a comparatively bright sky. At the time of observation the period of this pulsar was known, but its position was still uncertain by $\pm 10 \mathrm{~min}$ of arc, and it was fortunate that the diaphragm was placed accurately enough at the centre of the nebula to include the pulsar. Because of the strong interpulse the fundamental, at $30.22 \mathrm{~Hz}$, is weaker than the second harmonic which is aliassed and appears at $37.20 \mathrm{~Hz}(97.64-60.44)$.

Using this equipment at the Cambridge Observatories and at the Royal Greenwich Observatory on the Isaac Newton Telescope I have examined a number of supernova remnants, white dwarfs and other objects. The results are summarised in Table I. With the exceptions of Nova (DQ) Her 1934 and the nucleus of M 31 all the fluc-

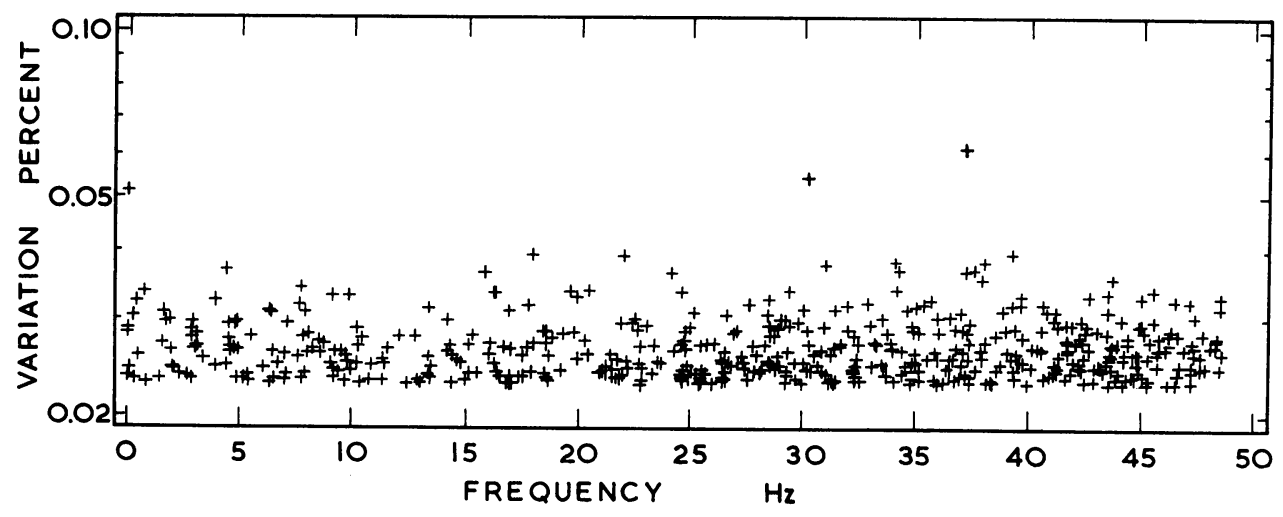

Fig. 2. NP 0532 observed on 24 November 1968. Data analysed after the discovery of optical flashes. This fast Fourier transform is based on 16384 readings made at $97.64 / \mathrm{sec}$ using the Cambridge $92 \mathrm{~cm}$ reflecting telescope with a diaphragm 50 arc s in diameter. The fundamental, at $30.22 \mathrm{~Hz}$, and the second harmonic, at $37.20 \mathrm{~Hz}$, are both present. 
tuations found were attributable to photon statistics or to irregular sky transparency. The observations of the nucleus of M 31, made on two different nights in 1969 August, showed a variation of the order of 0.15 per cent and with a period of 2.393 seconds. This was shown to originate in a periodic error in the drive of the telescope amounting to \pm 0.01 arc sec, which was caused by a slight eccentricity in one component of a small gearbox, rotating at 25 revolutions per sidereal minute. Such a small error is, of course, quite unimportant for other types of observation, and the fact that no variations were found at the frequency of rotation of the principal gears testifies to the work of the makers.

The limitations of this equipment are illustrated in Figure 3, which also indicates the observed limits of pulsed light from several pulsars. The upper limit to frequencies

\section{TABLE I}

Objects examined in search for regular fluctuations (other than NP 0532)

\begin{tabular}{|c|c|c|c|c|c|c|}
\hline Object & Date & Tel. & $\begin{array}{l}\text { Diaph. } \\
\text { arc sec. }\end{array}$ & Rate & $\begin{array}{l}\text { Max. var. } \\
\text { (per cent } \\
\text { or mag.) }\end{array}$ & $\begin{array}{l}\text { In frequency } \\
\text { range } \mathrm{Hz}\end{array}$ \\
\hline \multicolumn{7}{|l|}{ White dwarf stars } \\
\hline $\mathrm{GRW}+70^{\circ} 5824$ & 1969 May 16 & 98 & 13 & 97.6 & $\begin{array}{l}0.44 \\
0.21\end{array}$ & $\begin{array}{l}0.0-48.8 \\
0.025-8.1\end{array}$ \\
\hline W 1346 & 1969 Aug 14 & 98 & 13 & 48.8 & $\begin{array}{l}0.80 \\
0.48\end{array}$ & $\begin{array}{l}0.005-24.4 \\
0.005-8.1\end{array}$ \\
\hline R 627 & 1970 Mar 8 & 36 & 25 & 24.4 & $\begin{array}{l}0.64 \\
0.48 \\
0.21\end{array}$ & $\begin{array}{l}0.0-0.006 \\
0.006-12.2 \\
0.009-2.034\end{array}$ \\
\hline L $1244-26$ & 1970 Mar 8 & 36 & 13 & 12.2 & $\begin{array}{l}1.50 \\
0.33\end{array}$ & $\begin{array}{l}0.0-0.008 \\
0.008-6.1\end{array}$ \\
\hline L $1409-4$ & 1970 Mar 9 & 36 & 25 & 12.2 & $\begin{array}{l}1.30 \\
0.30\end{array}$ & $\begin{array}{l}0.0-0.012 \\
0.012-6.1\end{array}$ \\
\hline $\begin{array}{l}\text { Flare star } \\
\text { AD Leo }\end{array}$ & 1970 Mar 9 & 56 & 25 & 97.6 & 0.75 & $0.0-48.8$ \\
\hline Galaxies & & & & & & \\
\hline NGC 5548 & 1969 May 16 & 98 & 14 & 48.8 & $\begin{array}{l}0.67 \\
0.28\end{array}$ & $\begin{array}{l}0.006-24.4 \\
0.025-4.07\end{array}$ \\
\hline M 31 & 1969 Aug 14 & 98 & 7 & 48.8 & $\begin{array}{l}0.24 \\
0.14\end{array}$ & $\begin{array}{lr}1.3 & -24.4 \\
0.26 & -6.1\end{array}$ \\
\hline M 31 & 1969 Aug 18 & 98 & 7 & 12.2 & $\begin{array}{l}0.12 \\
0.10\end{array}$ & $\begin{array}{l}0.28-0.42 \\
0.42-1.525\end{array}$ \\
\hline NGC 4151 & $\begin{array}{l}1970 \text { Mar } 5 \\
1970 \text { Mar } 5\end{array}$ & $\begin{array}{l}36 \\
36\end{array}$ & $\begin{array}{l}25 \\
25\end{array}$ & $\begin{array}{l}97.6 \\
24.4\end{array}$ & $\begin{array}{l}0.60 \\
0.42 \\
0.26 \\
0.13\end{array}$ & $\begin{array}{l}0.0-48.8 \\
0.0-0.042 \\
0.042-12.2 \\
0.136-2.034\end{array}$ \\
\hline M 82 & $\begin{array}{l}1970 \text { Mar } 6 \\
1970 \text { Mar } 6\end{array}$ & $\begin{array}{l}36 \\
36\end{array}$ & $\begin{array}{l}50 \\
50\end{array}$ & $\begin{array}{l}97.6 \\
48.8\end{array}$ & $\begin{array}{l}0.40 \\
0.27 \\
0.10\end{array}$ & $\begin{array}{ll}0.9 & -48.8 \\
0.0 & -24.4 \\
0.023-4.068\end{array}$ \\
\hline M 87 & 1970 Mar 7 & 36 & 25 & 24.4 & $\begin{array}{l}0.27 \\
0.12\end{array}$ & $\begin{array}{l}0.062-12.2 \\
0.047-2.034\end{array}$ \\
\hline
\end{tabular}


Table I (Continued)

\begin{tabular}{|c|c|c|c|c|c|c|}
\hline Object & Date & Tel. & $\begin{array}{l}\text { Diaph. } \\
\text { arc sec. }\end{array}$ & Rate & $\begin{array}{l}\text { Max. var. } \\
\text { (per cent } \\
\text { or mag.) }\end{array}$ & $\begin{array}{l}\text { In frequency } \\
\text { range } \mathrm{Hz}\end{array}$ \\
\hline \multicolumn{7}{|c|}{ Planetary nebula } \\
\hline M 57 Nucleus & 1969 Aug 18 & 98 & 7 & 48.8 & $\begin{array}{l}1.50 \\
0.68\end{array}$ & $\begin{array}{l}0.0-24.4 \\
0.005-4.068\end{array}$ \\
\hline $\begin{array}{l}\text { Supernova remna } \\
\text { OA } 184 \\
\text { ( } 4 \text { areas searched) }\end{array}$ & $1970 \mathrm{Feb}$ & 36 & 50 & 97.6 & $16^{\mathrm{m}} \cdot 4$ & $\begin{array}{lll}0.0 & -48.8\end{array}$ \\
\hline $\begin{array}{l}\text { OA } 184 \\
\text { ( } 7 \text { areas searched) }\end{array}$ & $1970 \mathrm{Feb}$ & 36 & 240 & 97.6 & $13^{\mathrm{m}} .7$ & $0.0-48.8$ \\
\hline $\begin{array}{l}\text { IC } 443 \\
\text { ( } 2 \text { areas searched) }\end{array}$ & $1970 \mathrm{Feb}$ & 36 & 50 & 97.6 & $16^{\mathrm{m}} \cdot 7$ & $0.05-48.8$ \\
\hline $\begin{array}{l}\text { IC } 443 \\
\text { ( } 7 \text { areas searched) }\end{array}$ & $1970 \mathrm{Feb}$ & 36 & 240 & 97.6 & $13^{\mathrm{m}} \cdot 7$ & $0.0-48.8$ \\
\hline $\begin{array}{l}\text { HB } 9 \\
\text { (2 areas searched) }\end{array}$ & $1970 \mathrm{Feb}$ & 36 & 50 & 97.6 & $16^{\mathrm{m}} .5$ & $0.0-48.8$ \\
\hline \multicolumn{7}{|l|}{ Miscellaneous } \\
\hline $3 \mathrm{C} 273$ & $1970 \mathrm{Feb} 8$ & 36 & 25 & 97.6 & 4.00 & $\begin{array}{ll}0.0 & -48.8\end{array}$ \\
\hline BL Lac & 1969 Aug 17 & 98 & 13 & 24.4 & $\begin{array}{l}1.00 \\
0.56\end{array}$ & $\begin{array}{l}0.01-12.2 \\
0.20-2.03\end{array}$ \\
\hline & 1969 Aug 17 & 98 & 13 & 48.8 & $\begin{array}{l}0.68 \\
0.36\end{array}$ & $\begin{array}{l}0.015-24.4 \\
0.100-4.88\end{array}$ \\
\hline DQ Her & 1969 Aug 18 & 98 & 13 & 48.8 & $\begin{array}{l}0.88 \\
0.51\end{array}$ & $\begin{array}{l}0.01-24.4 \\
0.006-4.07\end{array}$ \\
\hline $3 C 386$ & 1969 Aug 18 & 98 & 13 & 48.8 & $\begin{array}{l}1.50 \\
0.64\end{array}$ & $\begin{array}{l}0.0-24.4 \\
0.008-4.07\end{array}$ \\
\hline
\end{tabular}

that can be determined unambiguously is half the rate of the readings, the Nyquist limit. Higher frequencies are detectable, with reduced sensitivity, and by using different recording rates in turn it is possible to resolve almost all ambiguities. The extreme lower limit to frequencies that can be observed is one cycle in the time covered by 16384 readings (the current limit of the computer and reduction programme). In practice random changes in sky transparency introduce noise at very low frequencies and there is usually some reduction in sensitivity. Over the rest of the frequency range the sensitivity is fairly accurately estimated by Equation (1), setting the duty cycle, $f=0.5$. For example, using the Isaac Newton Telescope with a diaphragm of 10 square seconds of arc and making 100 readings per second, the limit of detection should be about $22^{\mathrm{m}} .7$. If a pulsar similar to that in the Crab Nebula, which is apparently about 16.5, (Lynds et al., 1969), were located in Cas A, at twice the distance of the Crab and with 6 or 7 magnitudes of absorption instead of 1.7, it would have an apparent (integrated) magnitude of $22^{\mathrm{m}} \cdot 3$ or $23^{\mathrm{m}} \cdot 3$. But Cas A is only one third of the age of the $\mathrm{Crab}$, so it might be intrinsically much brighter. With such a small diaphragm it would take about 9 hours to search an area $20 \times 20$ arc sec. This search area covers only a small fraction of the area of the diffuse object, but so also did my original look at the Crab. 


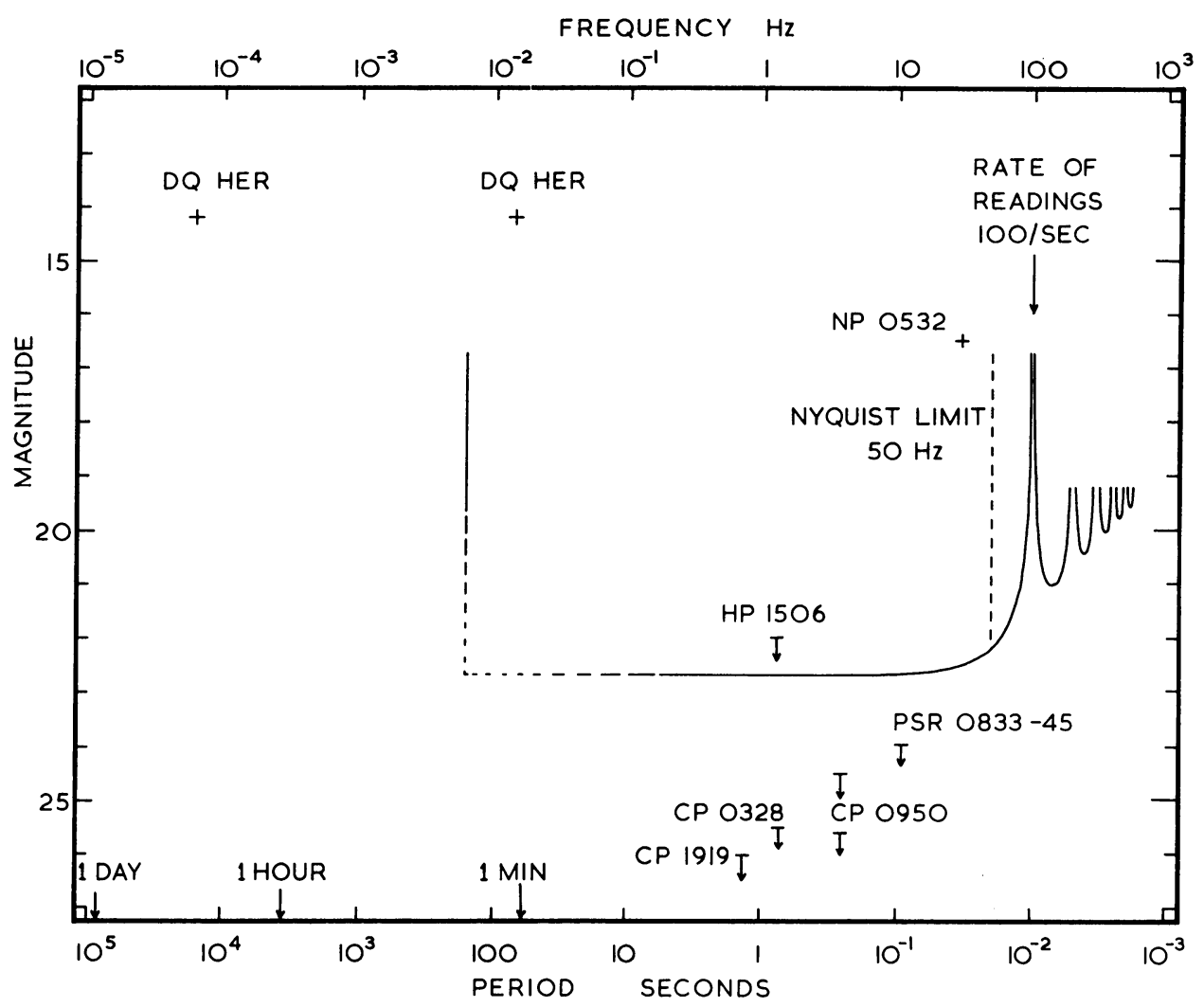

Fig. 3. Ordinates: Apparent magnitude; Abscissae: Frequency in $\mathrm{Hz}$ (top scale) or period in seconds (bottom scale). Symbols: + indicates the observed periodicities and time-average brightness of DQ Her (Walker, 1954, 1956) and NP 0532 (Lynds et al., 1969). I indicates the periodicities determined by radio observations and upper limits to the time average of any pulsed optical radiation from five other pulsars. See the text for details. The lines at $164 \mathrm{sec}$ period, and at $22^{\mathrm{m}} .7$ then curving up to $100 \mathrm{~Hz}$, indicate the limits of sensitivity of equipment built at Cambridge when used on the $249 \mathrm{~cm}$ (98-in.) Isaac Newton telescope with a photometer diaphragm 10 (arc sec) $)^{2}$ in area, and recording 16384 readings at $100 / \mathrm{sec}$.

So I conclude that the future of optical searches for pulsars is not hopeless, though the past has been less fruitful than some of us hoped.

\section{References}

Bahcall, J. N., Rees, M. J., and Salpeter, E. E.: 1970, Astrophys. J. 162, 737.

Cavaliere, A., Pacini, F.: 1970, Astrophys. J. 159, L21.

Cocke, W. J., Disney, M. J.: 1970, Bull. Am. Astron. Soc. 2, 190.

Lynds, R., Maran, S. P., Trumbo, D. E.: 1968, Science 161, 42.

Lynds, R., Maran, S. P., Trumbo, D. E.: 1969, Astrophys. J. 155, L121.

Reichley, P. E., Downs, G. S., and Morris, G. A.: 1970, Astrophys. J. 159, L35.

Walker, M. F.: 1954, Publ. Astron. Soc. Pacific 66, 230.

Walker, M. F.: 1956, Astrophys. J. 123, 68. 


\section{Discussion}

M. Rees: I would like to comment further on the calculations by Bahcall, Salpeter and myself which Dr. Willstrop has mentioned. We assume that the electromagnetic luminosity of a young pulsar can be obtained by scaling from the Crab pulsar according to the $\Omega^{4}$ law predicted by the electromagnetic dipole theory (which is pessimistic insofar as it implies that only $\sim 10^{-4}$ of the available energy is channeled into the optical band). Pulses should be detectable from a newly formed pulsar, spinning with its break-up angular velocity ( $\sim 50$ times the rotation speed of the Crab pulsar), out to distances $\sim 10 \mathrm{Mpc}$. The envelope would become transparent enough for optical pulses to shine through after 3 weeks to 3 months, depending on assumptions regarding the mass, velocity, and ionization of the expanding debris. The envelope would be more opaque to $\mathrm{X}$-rays and to radio pulses, so these would probably remain undetectable until the pulsar has slowed down substantially from its initial rotation rate. Optical observations thus seem to stand the best chance of detecting extragalactic pulsars.

We believe that these results are sufficiently encouraging to justify a search for optical pulses, with millisecond periods, from the sites of all newly-reported supernovae at distances $\leqslant 10 \mathrm{Mpc}$.

D. ter Haar: Calculations by Tsytovich, Buckee and ter Haar (Phys. Letters 32A, 1970) suggest that, of those pulsars for which $\mathrm{d} P / \mathrm{d} t$ is known apart from NP 0532, only PSR 0833 may pulse optically. Other pulsars may pulse in the infra-red. In these estimates we assume that the loss of rotational energy predominantly shows up at those frequencies where the brightness temperature is of the order of the effective temperature of the relativistic electrons. 